\title{
Morphologic Evidence of In-Situ Gold Deposition in Lateritic Placer Deposits from Guyana Shield of Venezuela
}

\author{
J. Silverstein ${ }^{1}$, M. Krekeler ${ }^{1}$ and J. Rakovan ${ }^{1}$ \\ 1. Department of Geology and Environmental Earth Science, Miami University, 250 South Patterson, \\ Ave., Oxford, Ohio
}

Recent studies have shown that gold can crystalize in-situ during diagenetic processes in fluvial or soil environments. In near surface environments gold is relatively insoluble, however, organic ligands such as humic acids can complex with gold and increase its solubility, providing a mechanism for gold precipitation in laterite (highly weathered soil) environments [1]. Electron microscopy is critical for observing micro-surface topology to identify unique textures and morphologies that suggest in-situ gold growth. A sample consisting of a gold nugget embedded in a laterite matrix from the Guyana shield of Venezuela was investigated to determine whether gold formed in-situ or by detrital processes. Scanning electron microscopy (SEM) shows gold textures within the laterite matrix that are commonly seen in organic rich soils at near surface conditions which suggest in-situ formation. Gold precipitation in porous regions found in the laterite show wire and fungoid-like morphologies, suggestive of biological precipitation (figure 1) [2,3]. Micro-surface topology of gold samples exhibits isolated nanometer to micrometer sized particles, agglomerated gold masses, and new gold layer growth, further suggesting insitu precipitation (figure 2).

SEM and energy dispersive spectroscopy (EDS) clearly shows a growth halo of discrete nanometer to micrometer scale gold particles adjacent to a gold nugget, along the surface, as well as in the pore spaces of the laterite material. The non-uniform size distribution of the gold in conjunction with well-formed crystal faces further suggests the gold particles were precipitated in-situ and not deposited mechanically. The exact mechanism of in-situ gold growth is unclear, however the results may have significant pragmatic implications. The occurrence of gold in lateritic placer deposits is common in Venezuela and samples from a variety of regions should be investigated to determine if the textures observed in this study are common in these settings. Nanometer and micrometer scale gold may not be recovered in traditional placer mining which is used in laterite ores, thus many sites may have waste or processed ore that has recoverable gold. Future work will focus on determining the prevalence of this texture in other Venezuelan samples and attempts to identify geochemical mechanisms for deposition.

References:

[1] AE Williams-Jones, RJ Bowell and AA Migdisov, Elements 5 (2009), p. 281.

[2] MF Lengke and G Southam, Geochemica et Cosmochinica Acta 70 (2006), p. 3646.

[3] L Fairbrother et al, Chemical Geology 320-321 (2012), p. 17 

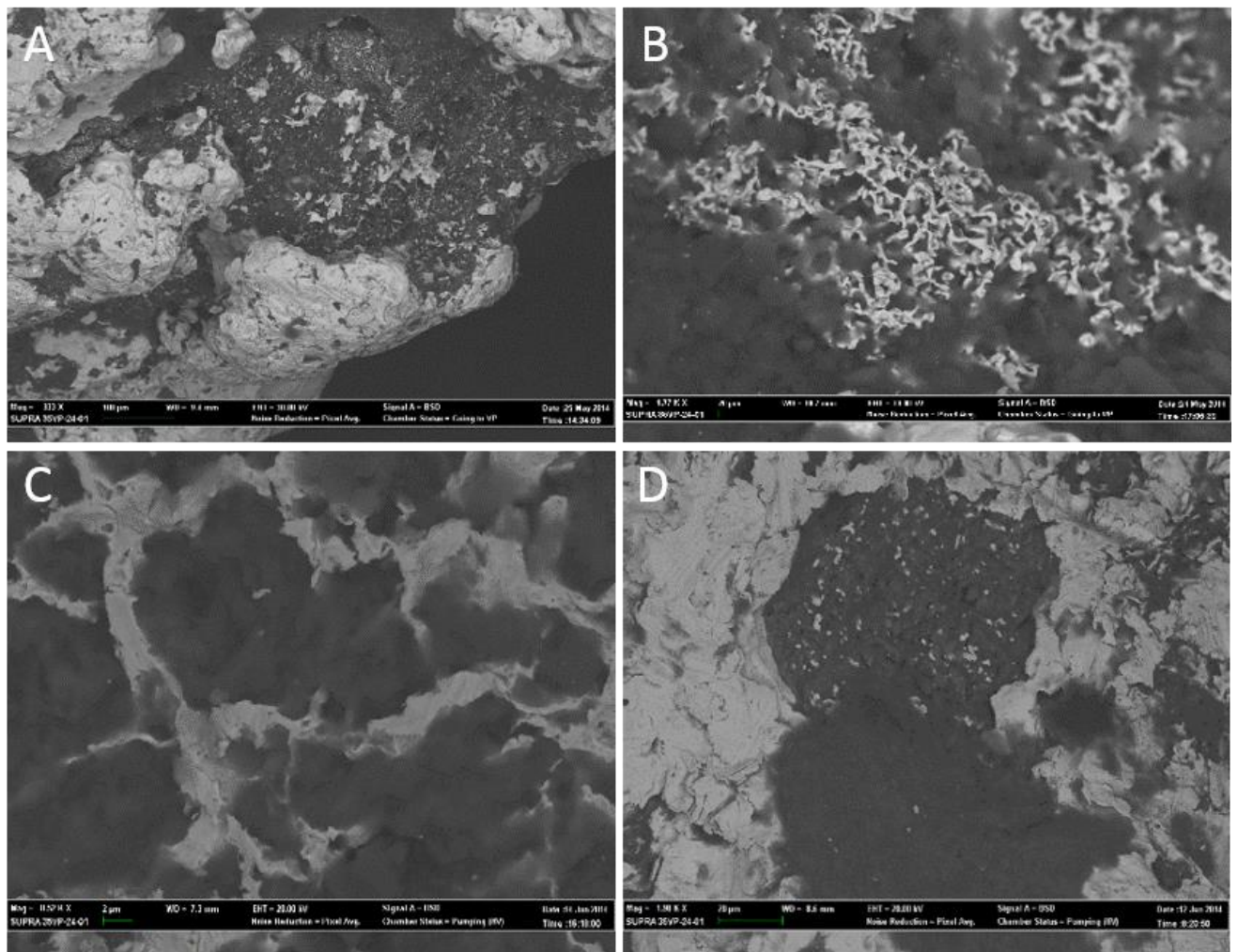

Figure 1. BSE micrographs showing the orientation of the gold leaflet masses existing within the porous networks of the surrounding soil medium. (A) Cavity in a large gold mass has a distribution of different gold particles with various morphologies. (B) Worm-like or fungoid texture from biological interactions; (C) Leaf or fin morphology that occurs in narrow channels within the soil medium; (D) Cavity in large gold mass showing gold growth where soil medium is of high porosity.

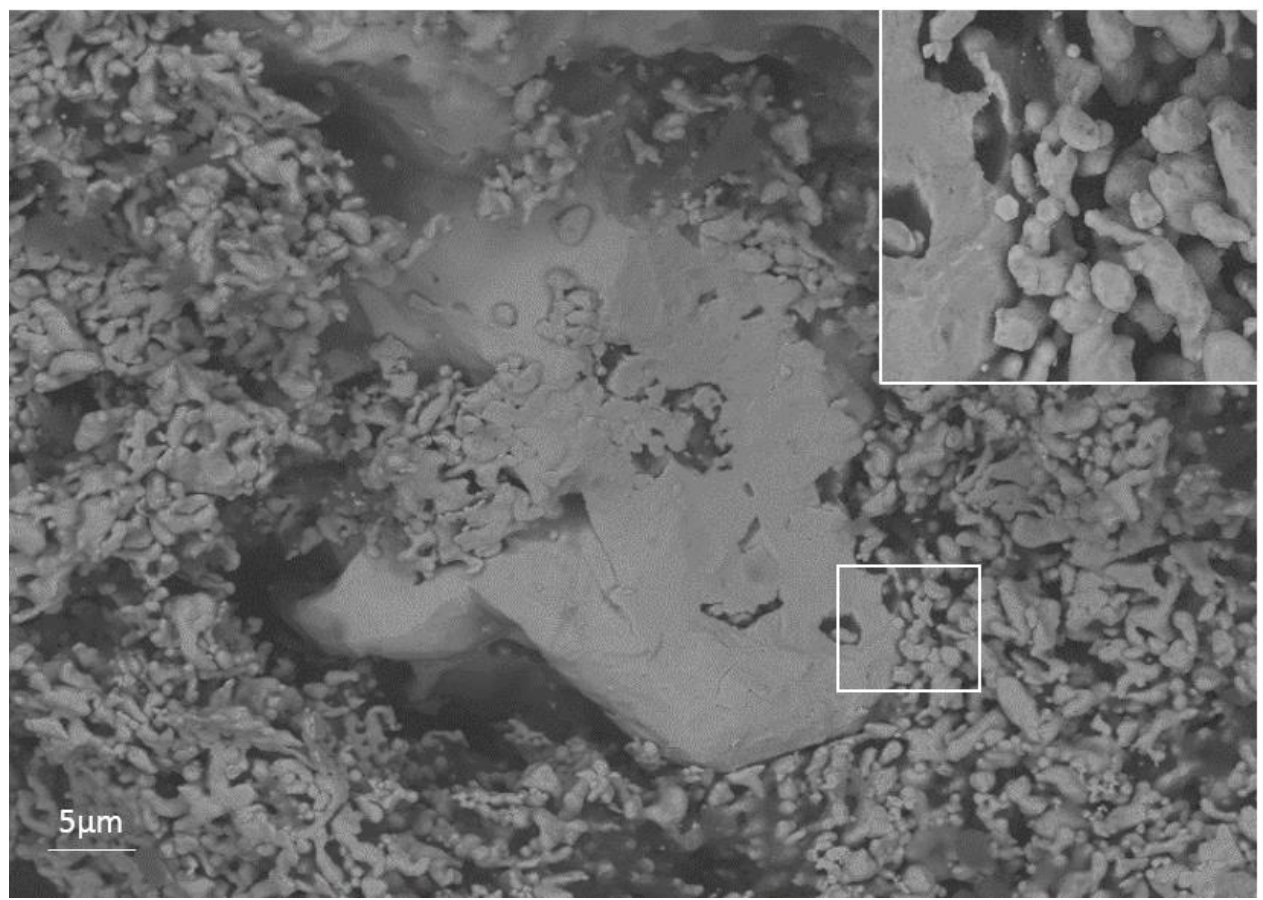

Figure 2. BSE micrographs of micro-surface topology of the gold surface and gold particles surrounding gold mass. (Inset) Euhedral gold crystals on the surface of the large mass, white box indicate location of inset micrograph 